\title{
On the performance of Planck-like telescopes versus mirror aperture
}

\author{
N. Mandolesi ${ }^{1}$, M. Bersanelli ${ }^{2}$, C. Burigana ${ }^{1}$, K.M. Górski ${ }^{3}$, E. Hivon ${ }^{4}$, D. Maino ${ }^{5}$, L. Valenziano ${ }^{1}$, F. Villa $^{1}$, and \\ M. White ${ }^{6}$ \\ 1 Istituto TeSRE, Consiglio Nazionale delle Ricerche, Via Gobetti 101, I-40129 Bologna, Italy \\ 2 IFC, Consiglio Nazionale delle Ricerche, Via Bassini 15, I-20133 Milano, Italy \\ 3 ESO, European Southern Observatory, Karl-Schwarzschild Str. 2, D-85748 Garching, Germany \\ ${ }^{4}$ CalTech, California Institute of Technology, 1200 East California Boulevard, Pasadena, CA 91125, U.S.A. \\ 5 SISSA, International School for Advanced Studies, Via Beirut 2-4, I-34014 Trieste, Italy \\ ${ }^{6}$ Harvard-Smithsonian Center for Astrophysics, 60 Garden St, Cambridge, MA 02138, U.S.A.
}

Received December 29, 1999; accepted April 26, 2000

\begin{abstract}
Future space mission like MAP and PLANCK will be able to shade new light on our knowledge of the Universe thanks to their unprecedented angular resolution and sensitivity. The far sub-degree angular resolution is obtained coupling usual detectors, radiometers and/or bolometers, to an optical system, namely a telescope. The wealth of cosmological information is encoded at high $\ell$ values $(\sim 1000)$ which can be reached with resolution of about $10^{\prime}$. Distortions of the main beam resulting from the current focal plane arrangement and the optical design of the PLANCK satellite will degrade angular resolution and sensitivity per resolution element possibly compromising the final results. The detailed design of the PLANCK telescope is continuously changing with the aim of optimizing its performance. In the present work we present a methodological study on the relation between telescope optical design, focal plane arrangement and optical performances, focussing on the dependence of angular resolution on primary mirror aperture. Different independent approaches have been developed to quantify the impact of main beam distortions on cosmic microwave background (CMB) science yielding nearly the same results. The socalled PHASE-A telescope is unacceptable with respect mission main goals. Larger telescopes (namely with effective aperture $\gtrsim 1.5 \mathrm{~m}$ ) are therefore preferable. This paper is based on the PLANCK LFI activities.
\end{abstract}

Key words: cosmology: cosmic microwave background methods: data analysis — telescopes

$\overline{\text { Send offprint requests to: }}$ mandolesi@tesre.bo.cnr.it

\section{Introduction}

Future space missions dedicated to the imaging of the cosmic microwave background (CMB), like PLANCK and MAP, represent a powerful opportunity for cosmology and astrophysics. The nominal angular resolution and sensitivity of the two instruments on-board PLANCK, the High Frequencies Instrument (HFI, Puget et al. 1998) and Low Frequencies Instrument (LFI, Mandolesi et al. 1998), allow to determine the angular power spectrum, $C_{\ell}$, of $\mathrm{CMB}$ primordial fluctuations up to multipoles, $\ell$, larger than $10^{3}$, i.e. until secondary anisotropies do not largely overwhelm primordial signatures. On the other hand, experiences from the previous CMB experiments as well as a realistic analysis of PLANCK observational performances indicate that a stringent control of all the systematic effects is crucial to reach the mission objectives (e.g. Burigana et al. 1998a,b; Delabrouille 1998; Maino et al. 1999; Maino 1999). Galactic and extragalactic foregrounds are important sources of astrophysical contaminations at a level that depend on the frequency $\nu$ and angular scale $\theta$ (e.g. Danese et al. 1996; Toffolatti et al. 1998), but, from the opposite point of view, their study represents an important co-product of the PLANCK mission (e.g. De Zotti et al. 1999 and references therein).

Two complementary approaches have been proposed by both LFI and HFI teams for reducing the impact of instrumental systematic effects on anisotropy measurements: the "hardware" approach, i.e. design mission strategy and instruments in order to minimize all the potential systematic effects, and the "software" approach, i.e. develop data analysis methods to further reduce residual effects in the data.

The detailed design of the PLANCK telescope is continuously changing with the aim of optimizing its 
performance. On the other hand, the optical framework and the basic concepts to address the relation between optical performances and scientific goals are quite general and possibly applicable also to other CMB anisotropy experiments. We present here a methodological study on the optical performance of the PLANCK-like telescopes, by comparing a suitable options of optical designs and analyzing their impact on the observations of CMB anisotropy measurements. After a brief discussion of the relative importance of the optical distortions near and far from the central direction of each beam in the sky field of view, we focus on the impact of main beam distortions on PLANCK data. Main beam distortions may introduce a degradation of the angular resolution and of the sensitivity per resolution element. These two last effects can be seen as orthogonal to each other in the space $\theta-\Delta T$ of angular scales and temperature anisotropy or, equivalently, in the space of $\ell-C_{\ell}$ (Mandolesi et al. 1997; Burigana et al. 1998a). The present analysis is devoted to recognize the "hardware" requirements of PLANCK-like telescopes in order to keep at acceptable levels the effects of main beam distortions.

In Sect. 2 we present a summary of the recent developments (Mandolesi et al. 1998; Puget et al. 1998) in the design of the PLANCK mission since the Phase A study (Bersanelli et al. 1996) focussing on the aspects relevant for the optical performance. In Sect. 3 we present the set of three of optical designs assumed as references for the present discussion, the basic framework of our optical simulations and our main results for the beam shapes; other optical configurations concerning telescopes with worst optical quality at the primary mirror edges are considered in Appendix A. We consider here the case of the "clean" $100 \mathrm{GHz}$ channels which are the most important for the primary cosmological goal, having small foreground contaminations. Moreover, in the new design of the Focal Plane Unit, the channels at highest frequencies of HFI are located very close to the telescope optical axis, where optical distortions are expected to decrease in order to compensate their increasing with the frequency. We present also a brief discussion of the optical performance at $30 \mathrm{GHz}$ in Appendix B. In Sect. 4 we estimate the implications of beam distortions by means of three different and complementary methods of analysis for quantifying the relevance of optical aberrations and by evaluating the final impact on $\mathrm{CMB}$ science. We set there the constraints to have a telescope "good enough" to reach the key goal of $\simeq 10^{\prime}$ resolution at $100 \mathrm{GHz}$. Section 5 concerns the limits on the edge taper for which the emission from the Solar System objects and from the Galaxy entering the sidelobes are acceptable without compromising the angular resolution. This is a first order analysis and therefore gives only an indication of the relevance of edge taper, sidelobes pick-up versus angular resolution. More detailed studies on the impact of sidelobes contamination have been done recently (e.g. De Maagt et al. 1998; Burigana et al. 1999b; Puget \& Delabrouille 1999; Wandelt \& Górski 1999). Finally, we discuss the mission impact of a such telescope, by dealing both with building problems and with cost problems, and draw out our main conclusions in Sect. 6 .

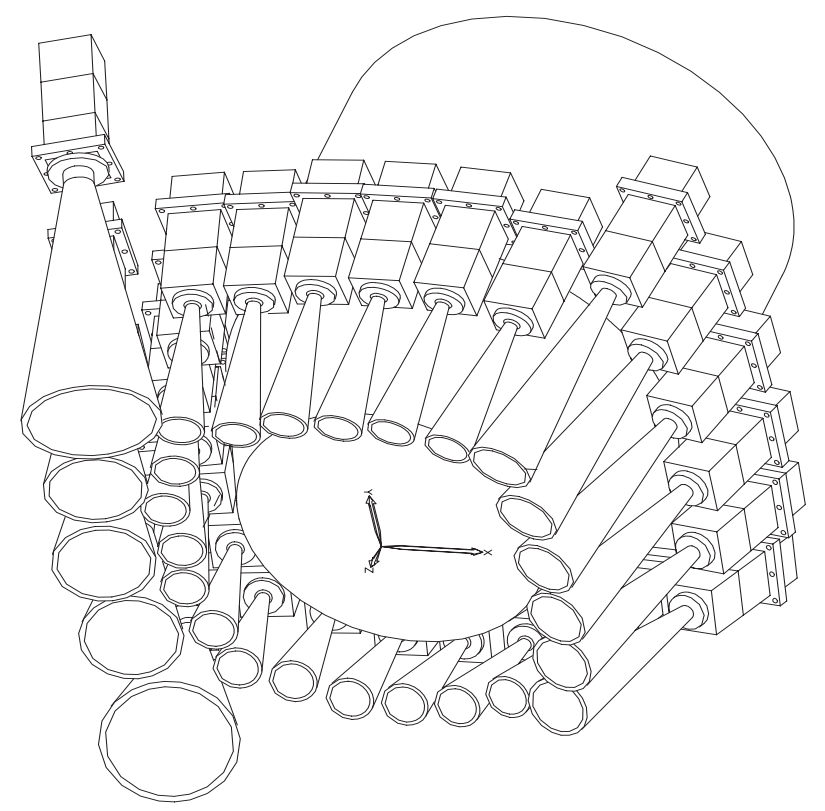

Fig. 1. New symmetric configuration accepted as current baseline (Mandolesi et al. 1998; Puget et al. 1998). The latter demands greater off-axis performance of the telescope due to the larger mean distance from optical axis for the LFI beams

\section{Recent developments relevant for the telescope design}

The baseline telescope design for PLANCK is similar to that originally proposed for COBRAS. The primary mirror is illuminated to maximize the angular resolution. An enclosing radiation shield controls the high sidelobe levels far off-axis that are the unavoidable result of the strong edge illumination. The system is a Dragone-Mizuguchi compensated optic and only the centre of the focal surface is aberration-free. The region of the focal surface where aberrations are acceptable from the point of view of angular resolution and beam shape has to be large enough to contain the feeds of a single instrument. With the merger of COBRAS and SAMBA into a single mission (Bersanelli et al. 1996) the demands placed on the telescope increased, because the region of the focal surface with small aberrations had to accommodate the feeds and cryostat from the HFI as well as the feeds from the LFI. During the Phase A study of the merged COBRAS/SAMBA concept the wavefront error at various locations in the focal surface was calculated and found to satisfy standard criteria for 
optical performances over a region large enough to accomodate feeds from two instruments.

Since the Phase A study there have been several developments with important implications for the telescope design. Detailed designs of the feed arrays in several configurations have been made by both PLANCK instrument teams, taking into account optical, mechanical, and thermal considerations. In the "asymmetric configuration" of the Focal Plane Unit (FPU) the LFI and HFI are accommodated side by side (Bersanelli et al. 1996). In the "symmetric configuration", preferred by both teams for mechanical and thermal reasons and recently specified as the baseline (Mandolesi et al. 1998; Puget et al. 1998), the HFI is centered on the optical axis and the LFI feeds are distributed in a ring around it (see Fig. 1), well outside the central region of the focal surface, where the optical performances are particularly critical. The study provided by TICRA (1997) on directivities and beam shapes at all relevant frequencies as well as those based on new optical codes developed by the PLANCK LFI team (Valenziano et al. 1998; Villa et al. 1997, 1998a,b) allowed to assess the impact of beam distortions on the scientific objectives of the mission. New techniques have been developed to evaluate the beam distortions specifically in terms of their effect on measurements of CMB anisotropies rather than by more conventional but less relevant criteria based only on optical properties (Mandolesi et al. 1997; Burigana et al. 1998a,b). This analysis, described in Sects. 3 and 4, shows that the standard rules of thumb for evaluating the performance of optical systems are not stringent enough for the demands of accurate CMB observations. As a meaningful example of the impact of the recent increasing of the theoretical understanding of the importance of angular resolution in CMB anisotropy observations, we observe how the NASA Midex mission MAP has increased the size of its two telescopes from $1.3 \times 1.5 \mathrm{~m}$ to $1.4 \times 1.6 \mathrm{~m}$ in order to improve the goal of its angular resolution from $18^{\prime}$ to $12^{\prime}$ at $90 \mathrm{GHz}$.

\section{Analyzed telescope designs and optical calculations}

We have studied the far sidelobe levels and main beam distortions for several different optical designs (see also Appendix A). On the other hand, in the following sections we will focus only on the same kind of PLANCK telescope design but for three different primary mirror aperture, for sake of simplicity and to give a better emphasis to the relevant issues.

The first (PHASE A) is the design presented in the Phase A study, a $1.3 \times 1.5 \mathrm{~m}$ off-axis Gregorian telescope (Bersanelli et al. 1996). The second (BASELINE) is an enlarged version of the Phase A design with a primary mirror of $1.550 \mathrm{~m}$ aperture, and presents an aperture very close to the present ESA baseline telescope. The third (ENLARGED) is an enlarged version of the Phase A

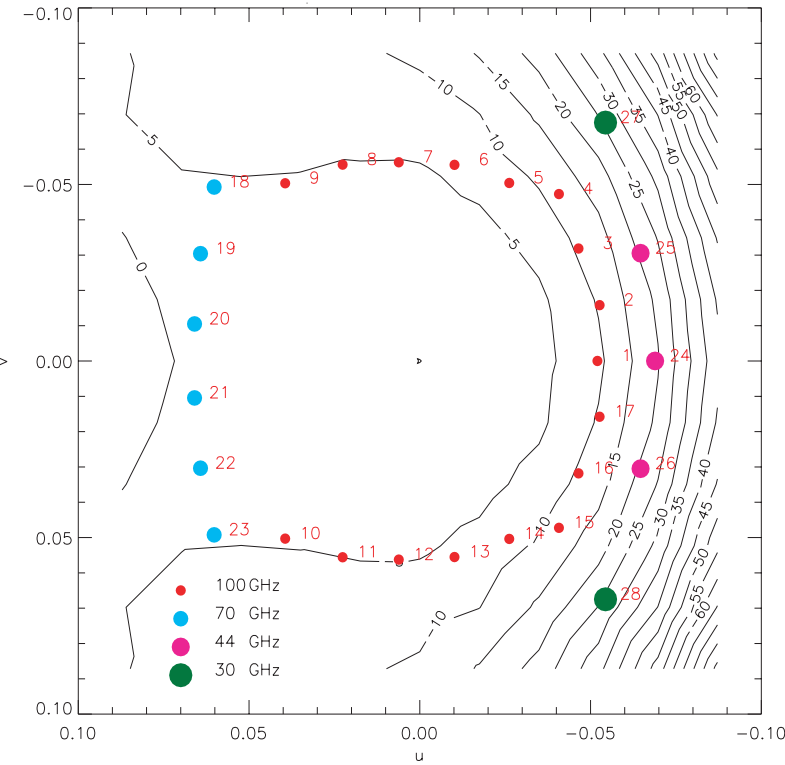

Fig. 2. Contour plot of the focal surface of the PHASE A telescope with the positions of the LFI feeds overlaid according to the symmetric configuration. The coordinates are $u \equiv \sin \Delta$ Elevation $\approx \Delta$ Elevation and $v \equiv \sin \Delta$ Azimuth $\approx \Delta$ Azimuth

design with a primary mirror of $1.750 \mathrm{~m}$ aperture. The overall focal ratio is the same for the three configurations: the incident wave is scattered by the primary parabolic mirror to the secondary ellipsoidal mirror and then to the focal region where the detectors are located. The secondary reflector axis is tilted at $14^{\circ}$ with respect to the parabola axis in order to minimize the beam distortions at the center of the focal region. Table 1 gives the details of the designs.

The properties of the radiation pattern have been calculated for the three optical configurations proposed for PLANCK telescope. For each of them, we calculated the position and the shape of the focal surface, i.e. the surface of maximum directivity (Valenziano et al. 1998). Geometrical optics approximation has been used: circular bundles of rays from the rim of the primary mirror are propagated through the telescope optics. The points where the bundles converge to the minimum size (in leastsquare sense) trace the focal surface and also give the correspondence between angles from the optical axis and the linear displacements in the focal surface, often referred as the plate scale. The longer effective focal lengths of the BASELINE and ENLARGED designs compared to the PHASE A design gives more magnified images (i.e., smaller plate scales in the sense that the number of arcminutes per millimeter is smaller), as shown in Figs. 2, 3 and 4 .

These results are used as input to calculate the response of the telescopes (the beam pattern) in a regular 
Table 1. Parameters of telescope designs analyzed

\begin{tabular}{ccccccc}
\hline Design & $D_{\mathrm{p}}(\mathrm{mm})$ & $F_{\mathrm{p}}(\mathrm{mm})$ & $2 a(\mathrm{~mm})$ & $2 c(\mathrm{~mm})$ & $d_{\mathrm{m}-\mathrm{s}}(\mathrm{mm})$ & $f_{\mathrm{eff}} / D$ \\
\hline PHASE A & 1292.4 & 720 & 1200 & 514.29 & 1065.69 & 1.29 \\
BASELINE & 1550.0 & 863.51 & 1200 & 514.29 & 1209.20 & 1.29 \\
ENLARGED & 1750.0 & 974.93 & 1200 & 514.29 & 1320.62 & 1.29 \\
\hline
\end{tabular}

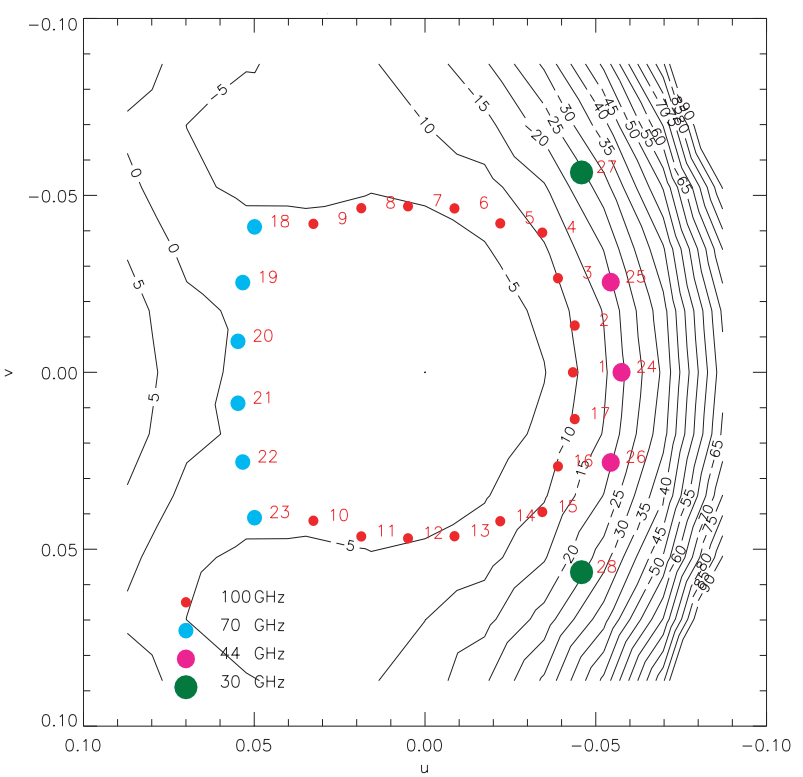

Fig. 3. The same as in Fig. 2 but for the BASELINE telescope. Note the difference in plate scale for the two telescopes

elevation-azimuth grid on the sky that covers the field of view (Villa et al. 1998b).

The detailed shapes of the main beams formed at each of the positions sampled on the sky were calculated using software based on Sletten (1988). Specifically, given the geometrical parameters of the optics, the far field is computed from the amplitude and phase distribution of currents on the main reflector surface. The amplitude is calculated by propagating the field from the horn to the main reflector, using geometrical scattering on the subreflector surface and taking into account the free space attenuation. The horn is modeled by a $\cos (\theta)^{N}$ amplitude beam pattern. The phase distribution is calculated by geometrical optics given the far field scan angle and the feed position. The repointing of the feed is systematically considered. The averaged distance between the subreflector and feeds is $75 \mathrm{~cm}$. The maximum diameter, $D_{\mathrm{f}}$, of LFI feeds is about $6.5 \lambda$ and the Fraunhofer region (Far Field) starts at about $2 \times D_{\mathrm{f}}^{2} / \lambda$ from the feed. This means that the Far Field distance is about $85 \mathrm{~cm}$ and $30 \mathrm{~cm}$ respectively at $30 \mathrm{GHz}$ and $100 \mathrm{GHz}$ so the Near Field effects are expected to be small and are neglected in this study.

The cross-check between our results and those by TICRA (1997), based on the full Physical Optics analysis with the GRASP8 code, shows good agreement both

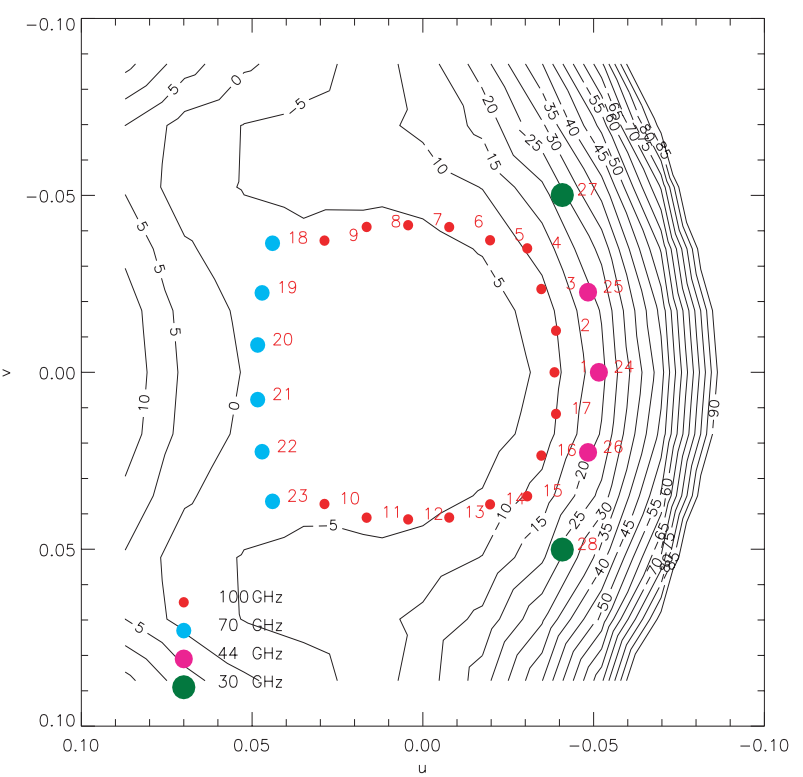

Fig. 4. The same as in Fig. 2 but for the ENLARGED telescope. Note the difference in plate scale for the two telescopes

for the predicted beam distortions, as shown in Fig. 5, and for the shape of the focal surface. All this validates the optical simulation codes considered here and allows us to use our simulated beams in the rest of the work.

\section{The effects of beam distortions on CMB science}

The critical question is to evaluate the effect of beam distortions on PLANCK science. In particular we want to know how the distorted beams affect the determination of the anisotropy power spectrum, and hence the estimation of cosmological parameters.

Standard criteria for the performance of optical systems do not directly address the study of the impact of beam distortions on CMB science. Because of this, we have developed three independent methods to characterize beam distortions and quantify their effect on $\mathrm{CMB}$ science. "A priori" they have somewhat different sensitivities to various types of beam distortions; nevertheless, they give similar answers, giving us confidence that we understand the effects of beam distortions.

We observe that the effect of main beam distortion in presence of foreground contamination should be in principle carefully considered. On the other hand, previous 

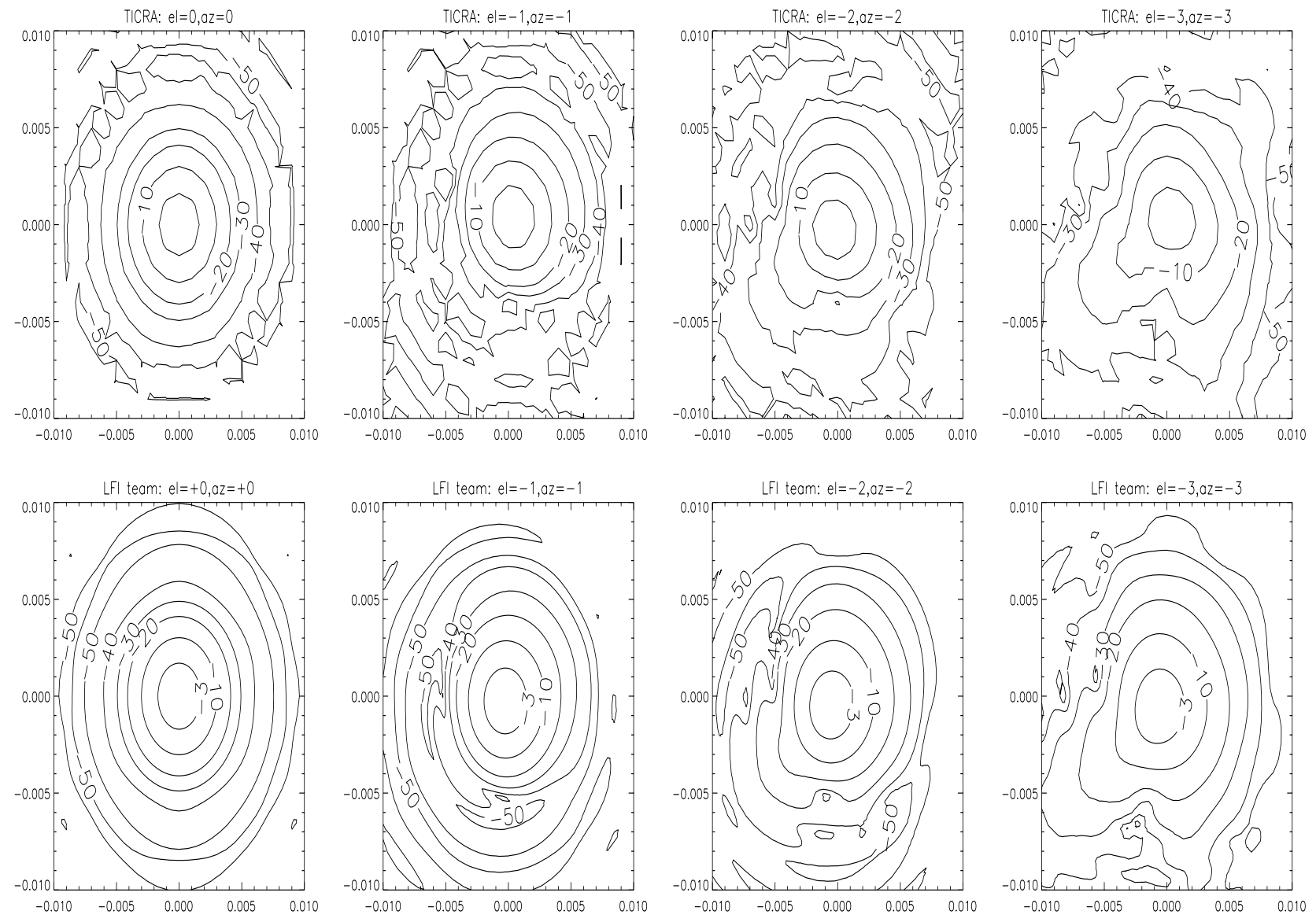

Fig. 5. Comparison of our simulated beams (labelled by "LFI team") with those computed by TICRA (1997) for the PHASE A configuration at $100 \mathrm{GHz}$

works confirm that the combination of these contaminations is not critical for PLANCK, at least for the LFI channels. Galaxy fluctuations combined to beam distortions produce a significant increase of the added noise (by a factor $\sim 3$ ) with respect to the case of a pure CMB sky only close to the galactic plane and at lowest frequencies (Burigana et al. 1998a). The combination of radiosource fluctuations and beam distortions produce in general only very small effects (Burigana et al. 1999a). Therefore, we consider here only the effect of the main beam distortions for a pure CMB fluctuation sky.

The methods presented below have been applied to the simulated beams computed by assuming an edge taper value of $30 \mathrm{~dB}$ (see Sect. 5 for details about the edge taper and its effects).

\subsection{Method 1: The effective window function}

In the first method an effective window function is calculated for the distorted beams and the loss in angular resolution from the corresponding rms temperature perturbations is then estimated.
The first step is to computing the spherical transform $w_{\ell m}$ for each of the beam response functions $w(\Omega)$ :

$w_{\ell m}=\int \mathrm{d} \Omega w(\Omega) Y_{\ell m}(\Omega)$,

with the beam centered at the pole of the coordinate frame. Then we simply consider the one-point variance of a distorted beam-smoothed temperature field:

$\left\langle\tilde{T}^{2}\right\rangle=\frac{1}{4 \pi} \sum_{\ell}(2 \ell+1) C_{\ell}\left[\frac{\sum_{m=-\ell}^{\ell} w_{\ell m}^{2}}{(2 \ell+1)}\right]$,

where the object in square brackets can be considered as a (Fourier space) symmetrized response function (labelled $\left.W^{2}(\ell)\right)$.

Plots of $W^{2}(\ell)$ computed for the beam patterns are shown for the PHASE A telescope in Fig. 6: the derived symmetrized Fourier beam shapes are not Gaussian. It is impossible to specify a simple, single, effective FWHM for such distorted beams. Instead, we show in the topleft panel of Fig. 6 the results of computations of the actual rms temperature perturbation in such beams from the usual CDM model (upper line) and the open CDM model (both roughly COBE-DMR normalized - but only relative 

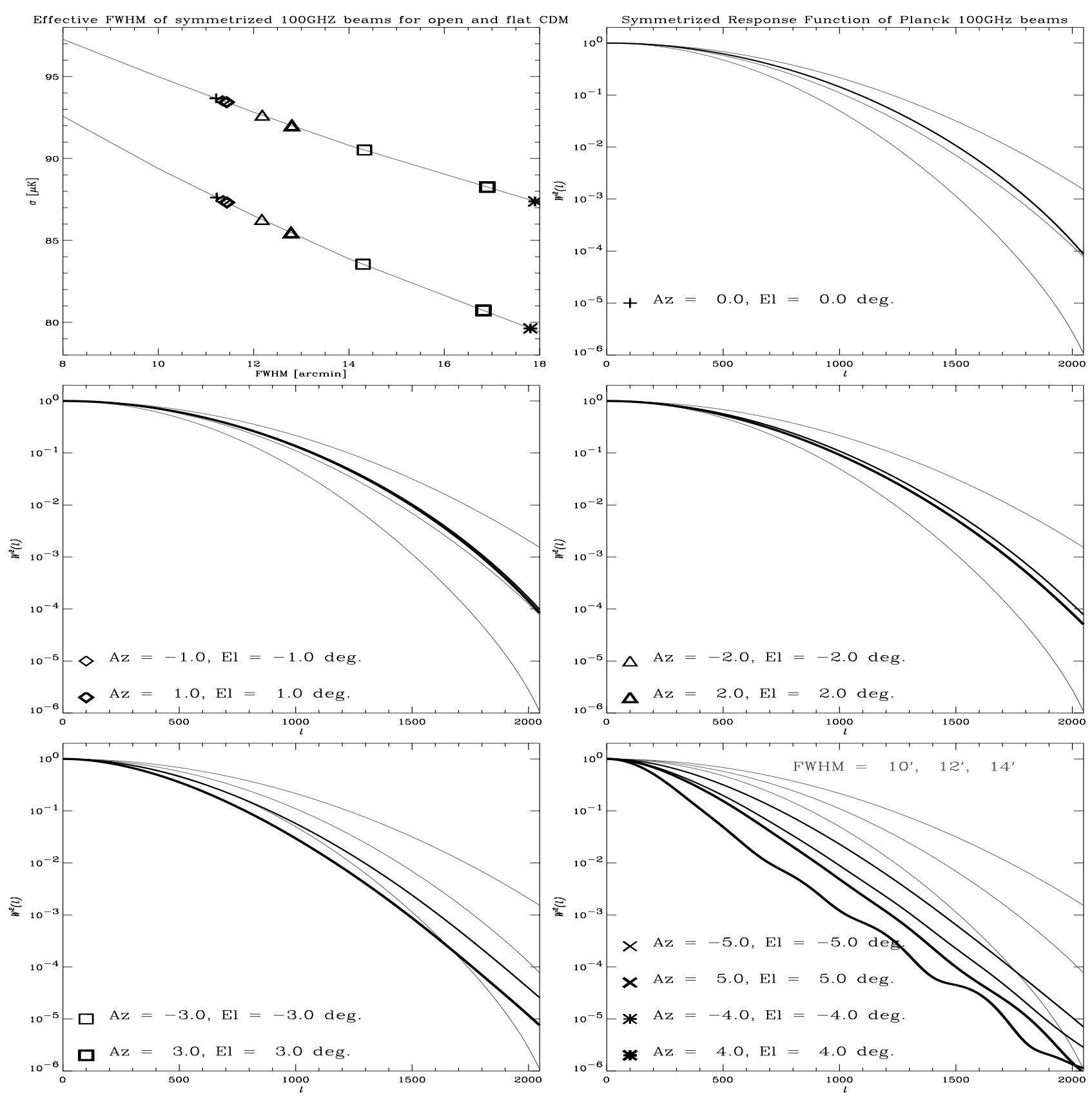

Fig. 6. Effective angular resolution of the PHASE A telescope calculated using Method 1. Upper right and bottom panelsComparison of calculated window functions (heavy lines) with the window functions of perfect Gaussian beams (i.e., $\left.\exp \left[-\ell(\ell+1) \sigma^{2}\right]\right)$ with $F W H M$ of $10^{\prime}, 12^{\prime}$, and $14^{\prime}$ (top to bottom, respectively). Labels inside the boxes give the offsets of the feed corresponding (top to bottom order) to each heavy line. The faster the window function falls off the broader the beam. The less Gaussian the window function the more distorted the beam. The two most off-set beam patterns $\left(A z=E l= \pm 5^{\circ}\right)$ are incompletely mapped in the provided data sets, and their corresponding Fourier transforms are odd, and incomplete. Top left panel - rms temperature differences that would be obtained with the actual distorted beams for the eleven feed positions show in the other panels, identified by symbol, for two difference CDM models (closed above, open below). The abscissa is the FWHM of the Gaussian beam that produces the same rms. A $100 \mathrm{GHz}$ feed on-axis would have an effective resolution of $11^{\prime}$ (with the assumed $30 \mathrm{~dB}$ edge taper). In the symmetric focal assembly configuration for the PHASE A telescope, however, the $100 \mathrm{GHz}$ LFI feeds are at a mean distance from the optical axis of $3.3^{\circ}$, i.e. $A z=E l= \pm 2.3^{\circ}$. The effective angular resolution of the PHASE A telescope for the LFI $100 \mathrm{GHz}$ feeds, therefore, is expected to be between $\simeq 12^{\prime}$ and $\simeq 15^{\prime}$. This is a serious degradation of the angular resolution 

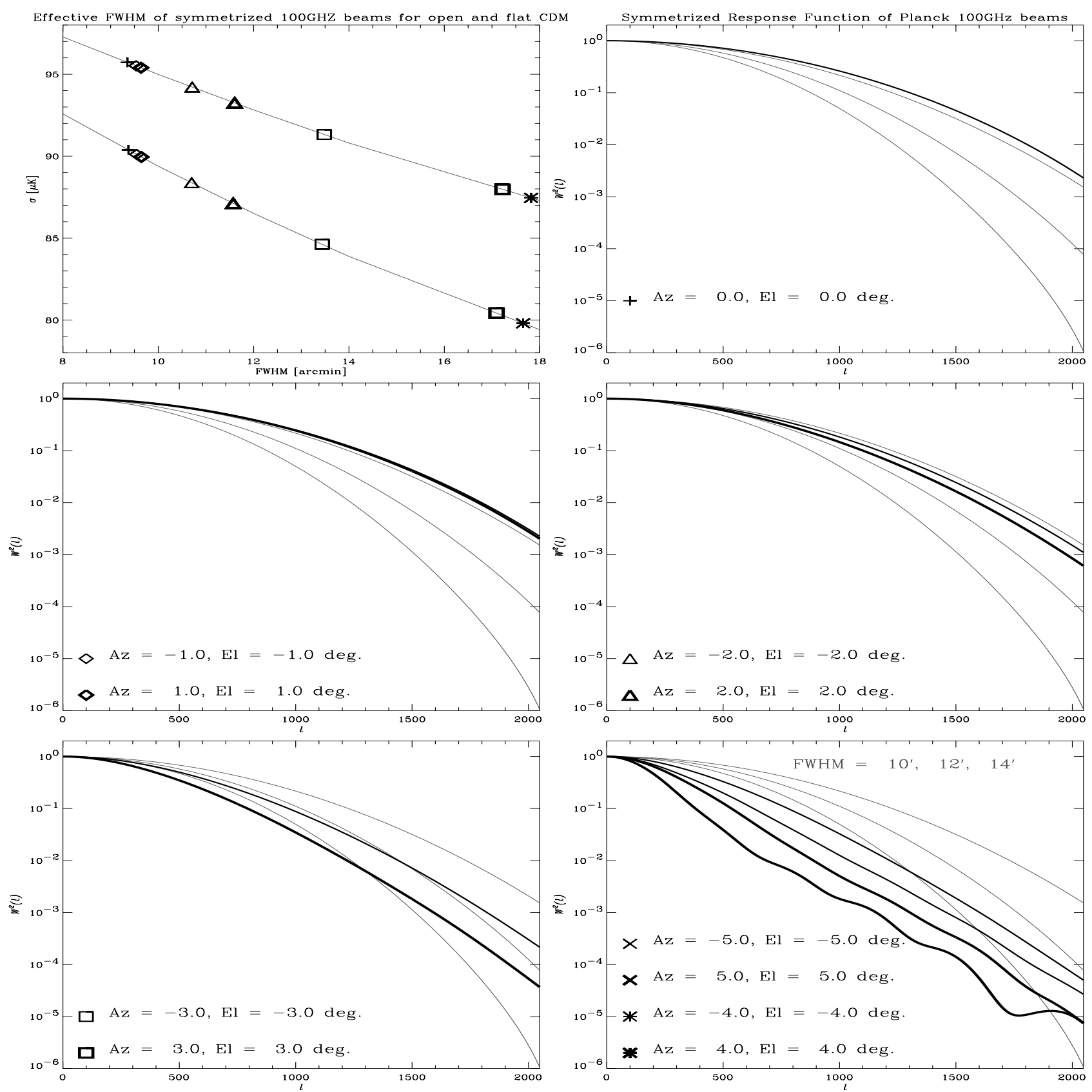

Fig. 7. The same as Fig. 6 (see the corresponding caption) but for the BASELINE configuration. This design is close to meet the goal of $\simeq 10^{\prime}$ resolution at $100 \mathrm{GHz}$

effects are relevant here). The curves show the rms temperature variation as a function of $F W H M$ of a symmetric Gaussian beam. Superposed symbols, to be matched with the other panels for beam identification, correspond to the actual values of rms temperature as measured by the distorted beams. From such a plot, within the context of a CMB anisotropy model, one can identify an effective angular resolution for non-symmetric beams. Note the symmetry of this effect in the $V$ direction and the large asymmetry in the $U$ direction which strictly reflects the intrinsic asymmetry of the adopted Gregorian configura- tion; the locations of the feedhorns in the focal plane have been designed to take this effect into account (see Villa et al. 1997 and Mandolesi et al. 1997, 1998). One can see that the PHASE A telescope has angular resolution poorer than $11^{\prime}$; the mean distance of $100 \mathrm{GHz}$ feeds from the optical axis is of $3.3^{\circ}$ or equivalently, for beams located along the "diagonals" $(|A z|=|E l|),|A z|=|E l|=2.3^{\circ}$ a value in the range $2^{\circ} \leq|A z|=|E l| \leq 3^{\circ}$ where the effective angular resolution of the PHASE A telescope ranges between a minimum value of $\simeq 12^{\prime}$ and a maximum value of $\simeq 17^{\prime}$. By avoiding the unfavourable locations 

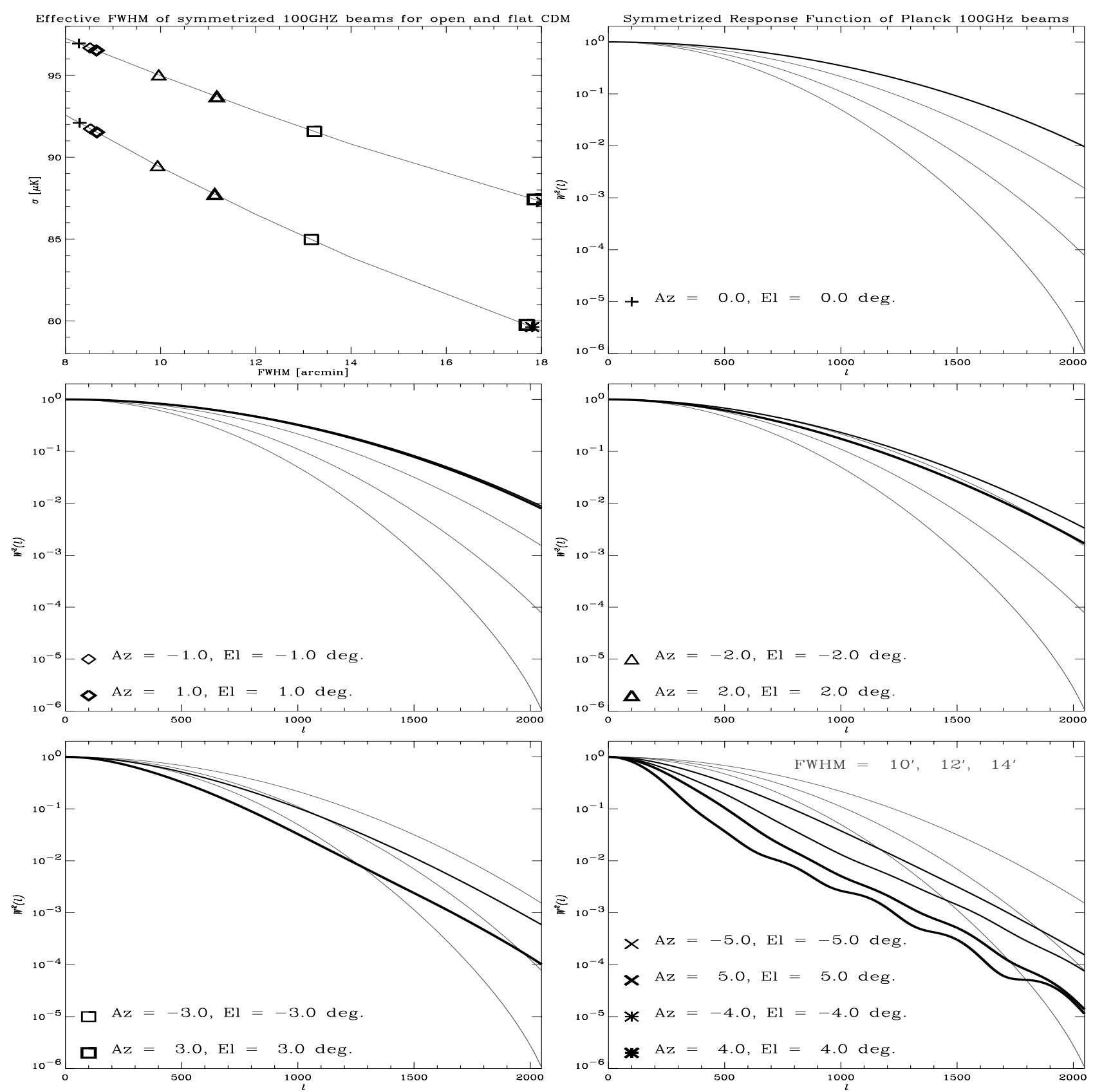

Fig. 8. The same as Fig. 6 (see the corresponding caption) but for the ENLARGED configuration. This design fully meets the goal of $\simeq 10^{\prime}$ resolution at $100 \mathrm{GHz}$

at $|A z|=|E l|$ with $E l>0$, the worst effective angular resolution reduces to $\simeq 14^{\prime} \div 15^{\prime}$, that still remains a serious degradation of the angular resolution.

Figures 7 and 8 show equivalent results for the BASELINE and ENLARGED telescopes. The significant improvement is due to the better optical performance as well as to the well known geometrical property that larger aperture telescopes lead to beam locations closer to the optical axis direction, so automatically releasing the issue of the distortions. Indeed, for these designs the mean offset of the LFI $100 \mathrm{GHz}$ feeds from the optical axis is respectively 2.8 and 2.5 . The BASELINE telescope is close to meet the $\simeq 10^{\prime}$ goal at $100 \mathrm{GHz}$ for all of the HFI and LFI feeds (see also the Table 3); of course, further improvements can be reached by the ENLARGED configuration.

\subsection{Method 2: SNR-weighted effective window functions}

In the second method observations are modeled by convolving the sky with the calculated beams and then adding noise. Specifically, the noise power spectrum is added 


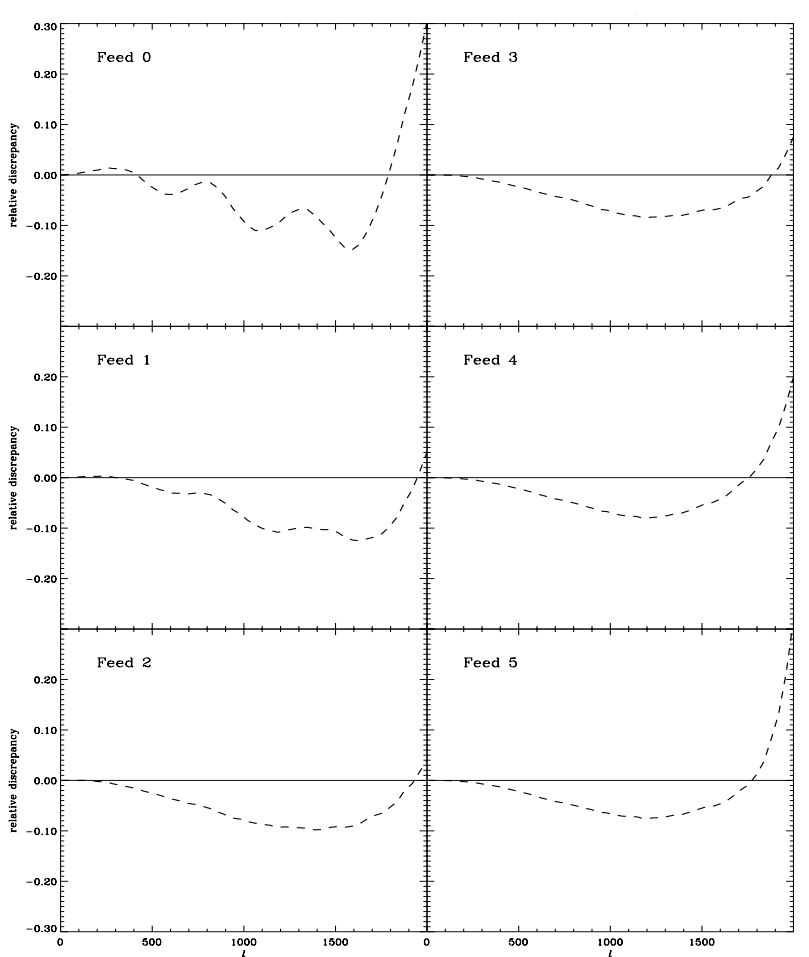

Fig. 9. Comparison of the results of Methods 1 and 2 for six feed positions. The differences are small except at very high $\ell$ values where the window functions are very small

to the product of the power spectrum of the sky signal and the window function. The window function is derived using a minimum variance estimator as described below (this requires use of a cosmological model, but the results are almost independent of the model assumed). Since the window function multiplies the signal, the optimum window function is as close to unity over as large a range of $\ell$ as possible.

For simplicity we approximate the sky as flat on the scales probed by the beam (i.e., $\sin \theta \approx \theta$; a very good approximation for beam sizes less than $1^{\circ}$ ). Then the spherical-harmonic transform becomes a Fourier transform and the 2-D window function is the (square of the) Fourier transform of the intensity of the beam. To ask what "equivalent" azimuthally symmetric beam corresponds to the 2-D window function thus computed requires a definition of what physically we mean by equivalent.

If we are concerned primarily with parameter estimation or reconstruction of the angular power spectrum, we can define "equivalent" to mean that which gives the same derivatives of the power spectrum with respect to the cosmological parameters (see Bond et al. 1997). Operationally this means a weighted sum of the $m$-modes which minimizes the variance. If $C(\ell)$ is our cosmological signal, $N(\ell)$ is the noise, and we approximate the sum over $m$ as an integral over $\theta_{\ell}$, then the optimal $W(\ell)$ solves:

$$
\left(1+\left[\frac{N}{C W}\right]_{\ell}\right)^{-1} \equiv \int \frac{\mathrm{d} \theta_{\ell}}{2 \pi}\left(1+\frac{N(\ell)}{C(\ell)} W^{-1}(\mathbf{l})\right)^{-1}
$$

where $W(\mathbf{l})$ is the 2-D window function and $\mathbf{l}$ is a $2-\mathrm{D}$ vector of length $\ell$. The only dependence on $\theta_{\ell}$ comes from the window function. In the angular average most of the weight at high $\ell$ comes from those parts of the beam that are narrowest. At low $\ell$, where the beam smearing is insignificant, all samples of the sky contribute equally. The criterion for keeping or suppressing directions in Fourier space is that the signal $\left(C_{\ell} W(\mathbf{l})\right)$ be measurable over the noise $\left(N_{\ell}\right)$.

Thus the "effective window function" depends on the signal-to-noise ratio, and so will be theory specific, but this dependence is very weak. In practice $W_{\ell}$ is well approximated by the quadrature mean of the two 1-D window functions obtained by slicing $W(\mathbf{l})$ in two orthogonal directions (i.e., $\sigma_{\text {eff }}^{2} \approx 1 / 2\left[\sigma_{x}^{2}+\sigma_{y}^{2}\right]$ ).

Although this calculation differs in detail from that of Method 1, the results are quite similar, as shown in Fig. 9.

\subsection{Method 3: Simulated observations}

In the third method a sky model (we assume a standard CDM model for the present tests) is numerically convolved with the calculated beams truncated at $1^{\circ}$ from the beam center, as well as with Gaussian beams of $F W H M$ from $6^{\prime}$ to $17^{\prime}$ in steps of $1^{\prime}$ (the integration uses a 2dimensional Gaussian quadrature with a typical grid of $48 \times 48$ points; see Burigana et al. 1998a for further details). All the beams are "artificially" relocated along the telescope optical axis and their centers observe the same set of positions in the sky; the different beam orientations in the sky related to the PLANCK scanning strategy is also taken into account.

We then calculate the rms of the difference between the convolutions obtained by using each simulated beam and Gaussian beams with increasing $F W H M$ : the Gaussian beam which minimizes the rms difference is considered as the "equivalent" Gaussian beam, which FWHM defines the effective angular resolution of the considered distorted beam. The values obtained with this method are in excellent agreement with those obtained with Methods 1 and 2. Figure 10 summarizes the results as a contour plot of effective angular resolution over the entire focal surface. Also, Table 3 gives the results of this method for the LFI feeds; we compute also the averaged rms differences (in terms of thermodynamic temperature), $\left\langle\sigma_{\mathrm{th}}\right\rangle$, between the convolutions from the simulated feeds and their corresponding equivalent Gaussian beams, that can be seen as an estimate of the additional error introduced by the beam distortion in absence of appropriate deconvolution techniques able to properly take into account the beam 

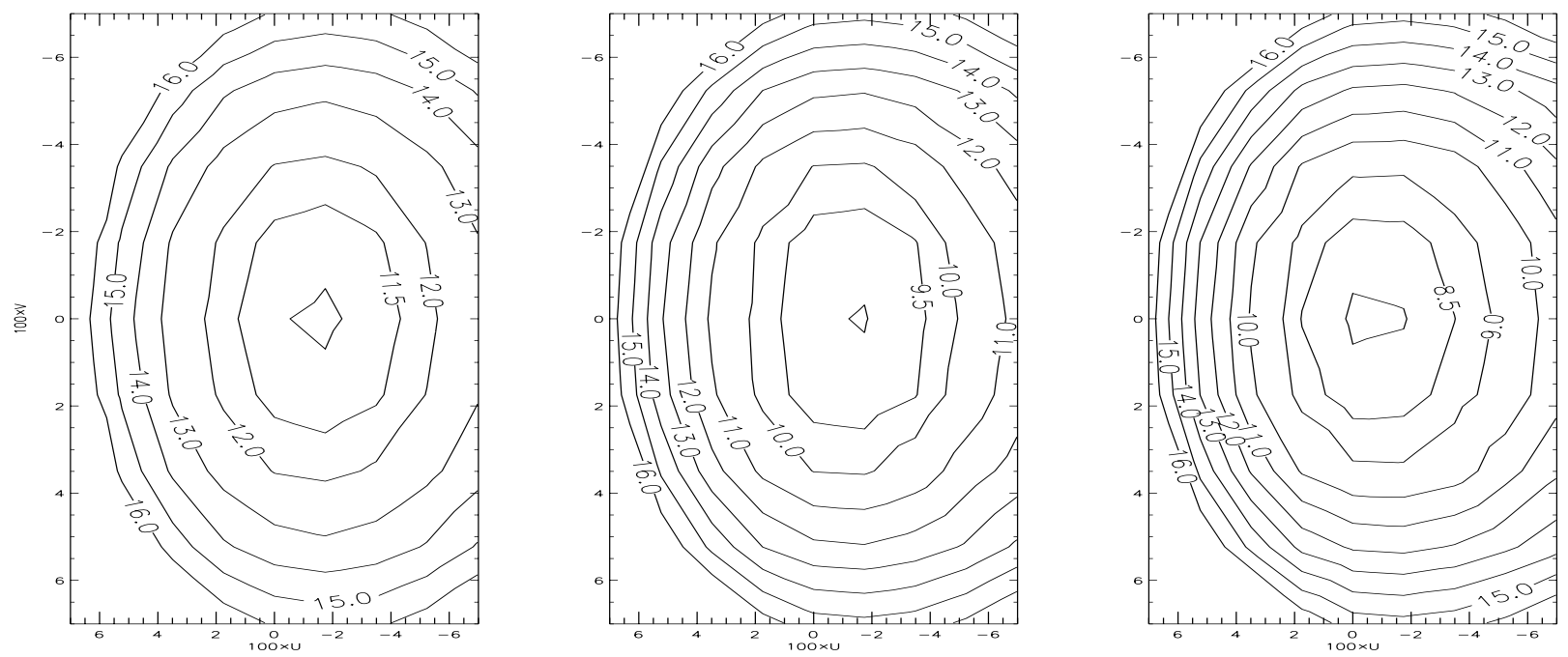

Fig. 10. Results of Method 3. Left panel: contours of effective angular resolution, given as the $F W H M$ of the symmetric Gaussian beam whose convolution with a CDM sky has the smallest rms difference with the convolution of the actual distorted beams of the PHASE A telescope. Central panel: the same for the BASELINE telescope. This design is close to meet the goals for angular resolution for both HFI and LFI feeds. Right panel: the same for the ENLARGED telescope. This design meets the goals for angular resolution for both HFI and LFI feeds

Table 2. Beam properties at $100 \mathrm{GHz}$ for telescopes of different aperture. We give the effective $F W H M$ (in arcmin), $W_{\mathrm{e}}$ : Results from pairs of beams located at the same $U$ and $|V|$ have been averaged. By averaging over all the feeds we have: $<W_{\mathrm{e}}>=13.1^{\prime}, 11.0^{\prime}, 9.7^{\prime}$ respectively for the $1.3,1.55$, $1.75 \mathrm{~m}$ aperture telescopes

\begin{tabular}{cccc}
\hline$D \rightarrow$ & $1.3 \mathrm{~m}$ & $1.55 \mathrm{~m}$ & $1.75 \mathrm{~m}$ \\
Feed & $W_{\mathrm{e}}$ & $W_{\mathrm{e}}$ & $W_{\mathrm{e}}$ \\
\hline 1 & 12.00 & 10.00 & 8.90 \\
2 \& 17 & 12.15 & 10.25 & 9.00 \\
3 \& 16 & 12.35 & 10.45 & 9.20 \\
$4 \& 15$ & 13.00 & 11.00 & 9.80 \\
5 \& 14 & 12.90 & 10.90 & 9.55 \\
6 \& 13 & 13.15 & 11.00 & 9.70 \\
$7 \& 12$ & 13.40 & 11.20 & 9.85 \\
8 \& 11 & 13.90 & 11.65 & 10.25 \\
$9 \& 10$ & 14.20 & 12.05 & 10.70 \\
\hline
\end{tabular}

shape: we find $\left\langle\sigma_{\mathrm{th}}\right\rangle=2.2,2.1,2.0 \mu \mathrm{K}$ for the PHASE A, BASELINE and ENLARGED design respectively.

As shown by Fig. 10 and Table 2, a larger telescope allows to reach the nominal $10^{\prime}$ resolution; this is due both to the better resolution of the best LFI feed and to the partial reduction of the (absolute) angular resolution degradation between the best and the worst LFI feed in the LFI ring region; unfortunately, increasing the primary mirror has a small impact on $\left\langle\sigma_{\mathrm{th}}\right\rangle$. This is due to the optical aberrations which are not reduced by scaling the telescope parameters.

This method gives also a particularly convenient way of quantifying one of the most important effects of distorted beams, namely, that data at the same position on the sky taken from multiple feeds at a given frequency cannot simply be averaged together, being each beam differently distorted, possibly with a different orientation (Burigana et al. 1998b).

By considering the set of data observed by Gaussian symmetric beams we have computed the differences in observed sky thermodynamic temperature, and the relative $\mathrm{rms}_{\mathrm{th}}$ values, between one beam and all the others and we have calculated that two Gaussian beams differing by $1^{\prime}$ yield $\mathrm{rms}_{\mathrm{th}}$ value of about $1.25 \mu \mathrm{K}$. Then the relation $\operatorname{rms}_{\mathrm{th}}(\mu \mathrm{K}) \sim 1.25 \Delta F W H M$ (arcmin) (solid line in Fig. 11) sets a lower limit to the rms of the temperature difference observed by distorted beams with a given difference $\triangle F W H M$ in effective resolution; the expected rms differences are larger due to asymmetries in the beam shapes, as shown in Fig. 11 for the PHASE A telescope.

Figure 12 gives a histogram of the differences between the signal measured at the same sky positions by the various LFI $100 \mathrm{GHz}$ feeds (solid lines) of the PHASE A and ENLARGED telescope for a suitable set of pointing directions. The mode of the distributions is $\simeq 3 \mu \mathrm{K}$, with some values exceeding $\simeq 5 \mu \mathrm{K}$. These values should be compared to the noise per beam $\sim 1 \mu \mathrm{K}$ that will be achieved by all PLANCK feeds up to $350 \mathrm{GHz}$ near the ecliptic poles. Note that this distribution is the superposition of the distributions of temperature differences obtained by comparing feeds with different spread in effective angular resolution; the central (peaked) part of this distribution (dotted lines) is dominated by the distributions concerning feeds with similar effective resolution whereas the distribution 


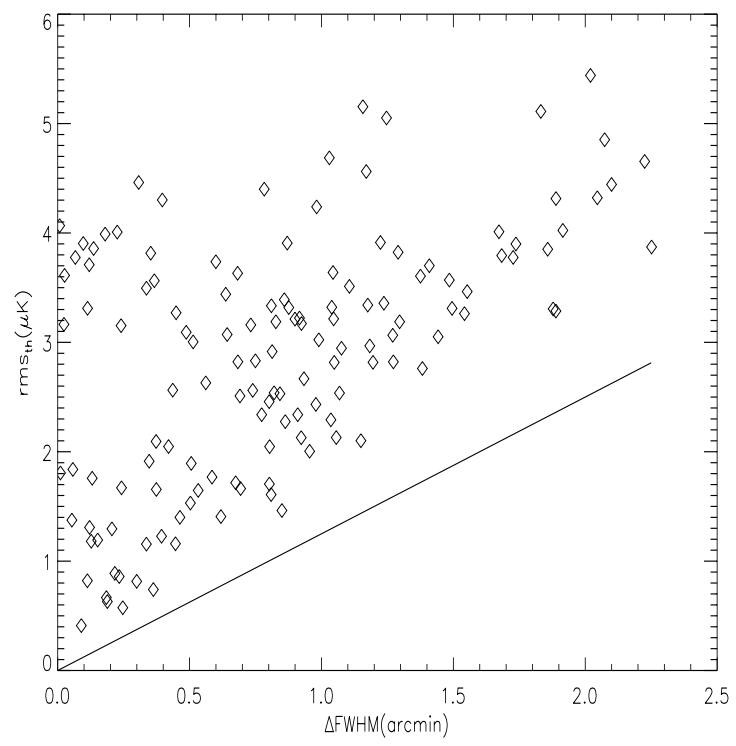

Fig. 11. Distribution of rms differences versus the difference of effective angular resolution for the PHASE A telescope; for the telecope designs in Table 1, this distribution is essentially independent of the telescope aperture (see the text for further details)

wings are dominated by the distributions concerning feeds with quite different effective resolutions (dashed lines).

Unfortunately, this dispersion of the sky temperature measurements cannot be significantly reduced by increasing the telescope aperture. It can be reduced only with a different FPU configuration which allows a location of all the $100 \mathrm{GHz}$ feeds closer to the centre or with a different telescope design able to redistribute the impact of optical aberrations in a more uniform way on the sky field of view (Villa et al. 1998a; Mandolesi et al. 1999) or, finally, in the data analysis.

No doubt some of these large effects can be removed in the data analysis, and it is certain that thousands of work-years will be devoted to the analysis of PLANCK data. Nevertheless, all previous experience with CMB data from ground, balloon, and space experiments shows that the more and the larger the systematic effects that must be scrubbed from the data in analysis, the more uncertain the result.

\subsection{Beam distortions and science}

The effects of degraded angular resolution on the scientific return from PLANCK have been estimated by calculating the uncertainties in cosmological parameters extracted from the angular power spectrum that would be obtained from the two telescope designs.
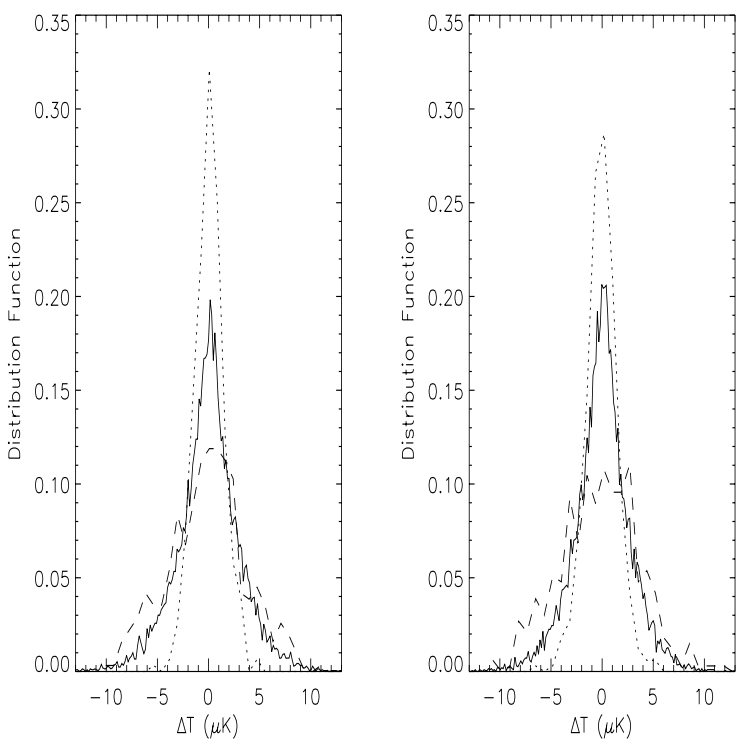

Fig. 12. Histograms of differences between the signal measured at the same sky positions by the $17 \mathrm{LFI} 100 \mathrm{GHz}$ feeds for a suitable set of pointing directions on a CDM sky, for the PHASE A telescope (left panel) and the ENLARGED telescope (right panel) (see the text for further details)

The fractional error on the CMB fluctuation angular power spectrum $C_{l}$ 's can be written summing in quadrature the cosmic variance and the instrumental noise (Knox 1995)

$\frac{\delta C_{\ell}}{C_{\ell}}=\left(\frac{4 \pi}{A}\right)^{1 / 2} \sqrt{\frac{2}{2 \ell+1}}\left[1+\frac{A \sigma^{2}}{N C_{\ell} W_{\ell}}\right]$

where $A$ is the surveyed area of the sky, $\sigma$ is the rms pixel noise, $N$ is the number of pixel in the sky map, $W_{l} \simeq \exp \left(-l^{2} F W H M_{\text {eff }}^{2} / 8 \ln 2\right)$ is a Gaussian approximation of the window function.

Of course, a detailed study, which is far from the purposes of the present work, requires the combined analysis of all the frequency channels and to take also into account the foreground contamination and an accurate quantification of the efficiency in the separation of the different components (e.g. Tegmark et al. 1999 and references therein) through Wiener filtering (Bouchet et al. 1995; Tegmark \& Efstathiou 1996), MEM (Hobson et al. 1999), wavelets (Sanz et al. 1999a,b; Tenorio et al. 1999) and independent component analysis (Bedini et al. 1999). On the other hand, we know that the "cosmologically clean" channels from 70 to $143-217 \mathrm{GHz}$ (according to the adopted evolutionary scheme for the galaxies in the far infrared) are minimally affected by foreground contamination at small scales and we consider their performance in absence of foreground contamination as a guideline for the discussion 
of impact of beam distortion on CMB science; moreover, from simple optical scaling laws and provided that we limit to the "cosmological" channels quite close to the $100 \mathrm{GHz}$ frequency, we expect that the effect of beam distortions in these channels is quite similar to that presented here at $100 \mathrm{GHz}$.

For high resolution and practically full sky experiments like PLANCK, the uncertainty on $C_{\ell}$ at large $\ell$ (larger than about $800-1000$ ) is dominated by the instrumental noise, which at any $\ell$ gives the error on our estimate of the observable realization of the $C_{\ell}$ 's. In this limit, given two experiment with angular resolution $F W H M_{1}$ and $F W H M_{2}$ respectively and the same sensitivity per pixel, the ratio between the uncertainty of the power spectrum recovered by the two experiments is given by

$\frac{\left(\delta C_{\ell}\right)_{1}}{\left(\delta C_{\ell}\right)_{2}} \simeq \exp \left[-\ell^{2}\left(F W H M_{2}^{2}-F W H M_{1}^{2}\right) / 8 \ln 2\right]$.

For the case of the PHASE A, BASELINE and ENLARGED telescopes the average $(F W H M)$ angular resolutions are $\simeq 13^{\prime}, 11^{\prime}$ and $9.7^{\prime}$ respectively; at multipoles $\ell \simeq 800,1000,1200$ and 1500 we find then $\left(\delta C_{\ell}\right)_{1} /\left(\delta C_{\ell}\right)_{2} \simeq 1.6,2.1,2.9$ and 5.2 or $\simeq 1.3,1.5,1.9$ and 2.7 by comparing PHASE A and BASELINE telescopes or BASELINE and ENLARGED telescopes, respectively.

It is then clear as the accurate determination of those cosmological parameters based on the accurate knowledge on CMB angular power spectrum at large $\ell$ is significantly affected by the quoted degradation of angular resolution introduced by the PHASE A telescope with respect to the PLANCK nominal goal of $10^{\prime}$ at $100 \mathrm{GHz}$. We consider here, as an example, an open (but not extreme) cold dark matter model like target model and use the standard Fisher matrix method for quantifying the impact of angular resolution degradation on PLANCK LFI observations. For simplicity, we use only the 70 and $100 \mathrm{GHz}$ channels together, neglecting foreground contamination. For the $70 \mathrm{GHz}$ beams we have not carried out detailed optical simulations, for the present purposes we simply estimate their averaged effective angular resolution by scaling our results at $100 \mathrm{GHz}$ according to two heuristic considerations: $i$ ) in absence of optical distortions, as for example very close to the optical axis, the beam $F W H M$ scales with $1 / \nu ; i i)$ for similar beam positions in the sky field of view (in the present FPU configuration the LFI feeds at 70 and $100 \mathrm{GHz}$ are located on a ring at approximately the same distance from the optical axis) the difference between the effective angular resolution and the nominal resolution along the optical axis quantifies the impact of optical aberrations and scales with $\nu$. At $70 \mathrm{GHz}$, we use then an effective $F W H M \simeq 17.7^{\prime}, 14.8^{\prime}, 13.1^{\prime}$ respectively for the PHASE A, BASELINE and ENLARGED telescopes. The results are given in Table 3 . From the optical simulations we know that aberration effects are not fully described by the degradation in effective angular resolution alone; they introduce also an additional, systematic noise which
Table 3. Uncertainties $(1-\sigma)$ in Extracted Cosmological Parameters versus Telescope Design. An OCDM model with $h=0.65, \Omega_{\mathrm{m}}=0.4, \Omega_{\mathrm{b}}=0.06, \Omega_{\Lambda}=0, \Omega_{\mathrm{K}}=0.6$ (curvature), $\tau=0.05, n_{\mathrm{S}}=1$ has been considered here as the fiducial model. We take $f_{\text {sky }}=0.65$

\begin{tabular}{cccc}
\hline Quantity & PHASE A & BASELINE & ENLARGED \\
\hline $\ln h$ & 0.0330 & 0.0248 & 0.0217 \\
$\ln \Omega_{\mathrm{K}}$ & 0.0377 & 0.0277 & 0.0232 \\
$\Omega_{\Lambda}$ & 0.0448 & 0.0327 & 0.0271 \\
$\ln \Omega_{\mathrm{b}} h^{2}$ & 0.0134 & 0.0125 & 0.0122 \\
$\Omega_{\mathrm{m}}$ & 0.0674 & 0.0493 & 0.0410 \\
$\ln n_{\mathrm{S}}$ & 0.0095 & 0.0089 & 0.0087 \\
$\ln \tau$ & 0.8074 & 0.7928 & 0.7870 \\
$\ln C_{2}$ & 0.0832 & 0.0815 & 0.0809 \\
\hline
\end{tabular}

could not be simplistically added in quadrature to the white noise; in particular, its angular power spectrum is not flat in $\ell-C_{\ell}$ space, as discussed in Burigana et al. (1999b). For the present purposes, we neglect here these additional effects focussing only on the impact of angular resolution: Table 3 should be then regarded as an optimistic assessment of the effects of beam distortions.

As known, the result partially depends on the choice of the set of parameters used in the analysis; on the other hand, this test confirms the results expected from a qualitatively point of view, i.e. the effect of degradation in angular resolution is particularly relevant for the determination of those parameters, as $h, \Omega_{\mathrm{m}}, \Omega_{\Lambda}$ and $\Omega_{\mathrm{K}}$, that show unambiguous signatures at large multipoles. Although for flat models this effect results to be less critical, we stress here that PLANCK is designed to be a third generation of $\mathrm{CMB}$ space mission and has to have the capability to disentangle between different cosmological models and to accurately determine the cosmological parameters for wide sets of cosmological scenarios and not only for some target models, by using separately each of the two instruments, for security and redundancy.

\section{Far sidelobes and beam resolution versus edge taper}

COBE had to deal with rapidly changing local conditions - temperature variations in the instrument and surroundings, changes in the position of the Sun, Earth, and Moon with respect to the instrument, varying magnetic fields, etc. Differential measurement techniques were required.

The PlanCK orbit, design, and scan strategy reduce most such sources of error by orders of magnitude. For example, the largest systematic error for the COBE DMR was modulation of the ferrite Dicke switches by the Earth's magnetic field. The magnetic field at $L_{2}$ is orders of magnitude smaller than experienced by COBE; moreover, for the LFI instrument the effect of system susceptibility to variations in the solar magnetic field will be well below $1 \mu \mathrm{K}$. 
Further, with the PLANCK spin configuration there is no first-order process to induce spin-synchronous thermal variations. In COBE these produced effects only at the $1 \mu \mathrm{K}$ level, and in PLANCK the effects will be still smaller. The extreme thermal stability at $L_{2}$ means that the time scale of thermal drifts will be long compared to the $60 \mathrm{~s}$ PLANCK spin period. The frequency dependence of the emissivity of the telescope surfaces and shields means that temperature stability requirements for PLANCK are driven by the HFI. For example, the HFI requirement for the temperature stability of the primary mirror is spinsynchronous variations of no more than $200 \mu \mathrm{K}$, compared to the LFI requirement of $1 \mathrm{mK}$ (assuming that the mirror emissivity is that of vacuum deposited aluminum).

The primary environmental sources of error for the LFI are those due to imperfect off-axis rejection by the optical system of radiation from the Sun, Earth, Moon, planets, and Galaxy. These are variations in response as the spacecraft spins that cause errors. Jupiter is the strongest compact source that can actually pass into the beam, and data must be corrected or excluded when it falls within the $\simeq-56 \mathrm{~dB}$ contour.

The radiation pattern at large angles from the main beam (sidelobes) is dominated by diffraction effects on the structure edges, that does not make negligible the response at large angles from the beam centre. Sidelobes introduce a contamination in sky temperature measured by the main beam due to the contribution of the sky signal entering the outer regions; this effect maybe significant principally owing to the Galactic emission, depending on the observed sky region, on the frequency and on the shielding efficiency. Further, the behaviour of the radiation pattern at intermediate angular scales from the beam centre has to be carefully considered.

The requirement on the rejection of radiation coming from directions far from the optical axis is stringent for PLANCK and does not pertain only to the telescope itself. Rather it is a requirement on the entire optical system, including the solar panel, shielding, telescope, and focal assembly components.

Since the far sidelobes of an antenna are largely determined by diffraction and scattering from the edges of the mirrors and from nearby supporting structures, they can be reduced by reducing the illumination of the edge of the primary. In the jargon of antenna design, this is called underilluminating the primary or, quantitatively, increasing edge taper, defined as the ratio of the power per unit area incident on the center of the mirror to that incident on the edge.

Of course, higher is the edge taper and lower is the sidelobe Galaxy contamination; on the other hand increasing the edge taper has a negative impact on the angular resolution. Results based on a simple computation performed in the parabola equivalent approximation are given in Table 4 for the LFI channels assuming beams located along the telescope optical axis. The spillover radiation
Table 4. Angular resolution, $F W H M$ (in arcmin), directivity (in $\mathrm{dB}$ ) and spillover losses at PLANCK LFI frequencies as function of the edge taper for the PHASE A Telescope. A simple parabola equivalent approximation has been used for these estimates that hold for beam located along optical axis only. The spillover has been calculated assuming a $\cos (\theta)^{N}$ feed pattern function: spill $_{\%}=100 \times 0.9278^{E T \cdot 3.074+1}$ where $E T$ is the edge taper in $\mathrm{dB}$

\begin{tabular}{ccccc}
\hline Edge Taper & $15 \mathrm{~dB}$ & $20 \mathrm{~dB}$ & $25 \mathrm{~dB}$ & $30 \mathrm{~dB}$ \\
\hline \multicolumn{5}{c}{$F W H M$} \\
\hline $30 \mathrm{GHz}$ & 32.5 & 34.6 & 36.8 & 39.0 \\
$44 \mathrm{GHz}$ & 22.2 & 23.6 & 25.1 & 26.6 \\
$70 \mathrm{GHz}$ & 13.9 & 14.8 & 15.8 & 16.7 \\
$100 \mathrm{GHz}$ & 9.8 & 10.4 & 11.0 & 11.7 \\
\hline \multicolumn{5}{c}{ Directivity } \\
\hline $30 \mathrm{GHz}$ & 51.1 & 50.6 & 50.0 & 49.5 \\
$44 \mathrm{GHz}$ & 54.4 & 53.9 & 53.4 & 52.8 \\
$70 \mathrm{GHz}$ & 58.4 & 58.0 & 57.4 & 56.8 \\
$100 \mathrm{GHz}$ & 61.5 & 61.1 & 60.5 & 59.9 \\
\hline \multicolumn{5}{c}{ Spillover \% } \\
\hline \multicolumn{5}{c}{0.9} \\
\hline
\end{tabular}

is calculated as a ratio between the power outside the reflector and the total power emitted by the feed.

Sidelobe structure sweeping across the Galaxy can produce artifacts in any direction, particularly, for LFI, at the lowest frequencies. From numerical simulations based on the optical results of De Maagt et al. (1998), objects of detailed works (e.g. De Maagt et al. 1998; Burigana et al. 1999b; Puget \& Delabrouille 1999; Wandelt \& Górski 1999), we find that the Galaxy straylight contamination at $30 \mathrm{GHz}$, defined as the signal entering from $\sim 1^{\circ} \div 2^{\circ}$ from the beam centre, shows a peak level at about $13 \mu \mathrm{K}$ at low galactic latitudes, to be principally ascribed to the beam response at few degrees to the main beam, and maximum values of about $6 \mu \mathrm{K}$ at high galactic latitudes, to be principally ascribed to the radiation entering the main spillover at $\simeq 90^{\circ}$ from the beam centre; the latter effect may be particular significant, contaminating the sky regions clean from relevant Galaxy contributions.

The PLANCK data will significantly improve our knowledge of galactic emission. Given a good characterization of the far sidelobes of the optical system before launch, a self-consistent reconstruction of both the Galaxy and the telescope radiation pattern could be made. But there will be residual errors in the reconstruction, and the purpose of PLANCK is to image the CMB, not the Galaxy. To ensure that the residual errors do not compromise PLANCK's primary science, we require that the level of galactic contamination be below the white noise level at $100 \mathrm{GHz}$ for 12 months of observations, with a factor of two margin to allow for uncertainties in the level of galactic emission, over $50 \%$ of the sky. This can be translated into a requirement on the sidelobe levels outside the central beam, calculated for the actual positions of the feeds. The exact 
number depends on the details of the sidelobe pattern, but is in the range -60 to $-70 \mathrm{~dB}$.

\section{Conclusions}

We have developed optical calculation codes to compute the focal surface, optimize feedhorn positions and calculate the main beam patterns on the focal surface of a suitable set of PLANCK-like telescopes. In particular we focussed on off-axis Gregorian telescopes with three different primary mirror apertures possibly including the socalled baffle option. Main beam patterns have been found to be mainly affected by spherical aberration and coma. Our results are in good agreement with those of TICRA (1997) based on the GRASP8 code and provide an adequate base for studying the impact of optical distortions on PLANCK science.

Three different independent methods of analysis have been proposed to quantify the optical distortion impact on Planck observations. The first two (semi-analytical) methods provide fast and accurate evaluations of the angular resolution degradation of PLANCK beams for different locations on the focal plane. The third (fully numerical) method also allows to quote the amount of additional noise introduced by main beam distortions with respect to Gaussian beams equivalent from the point of view of angular resolution. For what concerns the effective angular resolution, the three approaches are in very good agreement, thus confirming the reliability of our methods. For PLANCK-LFI beam locations on the focal surfaces of $1.3,1.55$ and $1.75 \mathrm{~m}$ telescopes we found averaged effective angular resolution $(F W H M)$ of $\left\langle W_{\mathrm{e}}\right\rangle \simeq 13.1^{\prime}, 11.0^{\prime}, 9.7^{\prime}$ and averaged rms increased noise $\left\langle\sigma_{\mathrm{th}}\right\rangle \simeq 2.2,2.1,2.0 \mu \mathrm{K}$ respectively.

By using the standard formula for $C_{\ell}$ uncertainty in CMB experiment, we provided simple estimates of the angular resolution degradation effect on the recovered $\mathrm{CMB}$ power spectrum at large $\ell$. The Fisher Matrix method has been implemented to compute the LFI uncertainties on the estimation of a suitable set of cosmological parameters as a function of the telescope aperture. As expected, a larger aperture and the resulting better resolution is particularly important for recovering cosmological parameters which show unambiguously imprints at large multipoles. This is particularly crucial for open models where all the relevant cosmological information encoded into the Doppler peaks is shifted towards large multipoles. As an example the error on $\Omega_{\Lambda}$ decreases from $4.5 \%$ to $2.7 \%$ by increasing telescope aperture from $1.3 \mathrm{~m}$ to $1.75 \mathrm{~m}$. Present results have to be considered as lower limits of the impact of main beam distortions since only the resolution degradation and not the additional noise has been considered. Furthermore the straylight contamination, which has not been included here, may increase the overall uncertainty, mainly affecting the low $\ell$ part of the power spectrum, and then just those cosmological parameters practically unaffected by main beam distortions. We stress here that both LFI and HFI on-board PLANCK satellite, a third generation of CMB space missions, are going to be designed to accurately recover the CMB anisotropy pattern for whole set of cosmological models not ruled out by present data limited only by astrophysical contaminations.

From the above results, it is clear that a telescope with an aperture $\gtrsim 1.7 \mathrm{~m}$ has to be regarded as a preferable choice to fully achieve PLANCK-LFI goals. On the other hand, the actual $\simeq 1.5 \mathrm{~m}$ baseline telescope results to be a reasonable compromise between the overall mission constraints and the scientific goals. The BASELINE telescope aperture is a significant improvement with respect to the PHASE A telescope, providing an angular resolution quite close to the LFI goal and allowing for an optimized tradeoff between main beam distortion and sidelobe effects. From the encouraging studies of Villa et al. (1998a) and Mandolesi et al. (1999), it results that an Aplanatic telescope design shows significant improvements with respect to the regularity of the beam shapes (quite close to ellipses with typical axes ratios of $\sim 1.2$ ) as well as to the uniformity of the beam angular resolution on the focal surface. This kind of designs is actually the considered baseline for the industrial studies by ALCATEL for the optimization of the PLANCK telescope in order to fully achieve the scientific goals.

Acknowledgements. It is a pleasure to thank T. Cafferty, M. Dragovan, P. Guzzi, V. Jamnejad, J.-M. La Marre, A. Lange, C. Lawrence, M. Malaspina and L. Wade for useful discussions.

\section{Appendix A: On the so-called "baffle" options}

The use of a properly designed reflective ring-baffle around the primary mirror, proposed to meet the edge taper requirement, and a minor modification in the design of the feeds (which will depend on their precise location in the focal plane) allow to obtain the illumination required to recover the PLANCK angular resolution.

We have carried out a second set of simulated beam patterns, for non-standard telescopes, assuming telescope configurations as listed in Table 5 and keeping the $30 \mathrm{~dB}$ edge taper requirement for all the channels.

A decisive comparison can be made in terms of equivalent angular resolution.

The results achieved with a system which includes the ring-baffle can be understood using the Method 1 and by comparing the plots of Figs. 13 and 14 with those of Fig. 6 : only up to $\sim 1.5^{\circ}$ of scan angle a reduction of the magnitude of angular degradation can be reached. The second and third - left and right - panels from the top in Fig. 15 show the results of Method 3 for these two configurations and compare them with those holding for the three configurations of Table 1. 

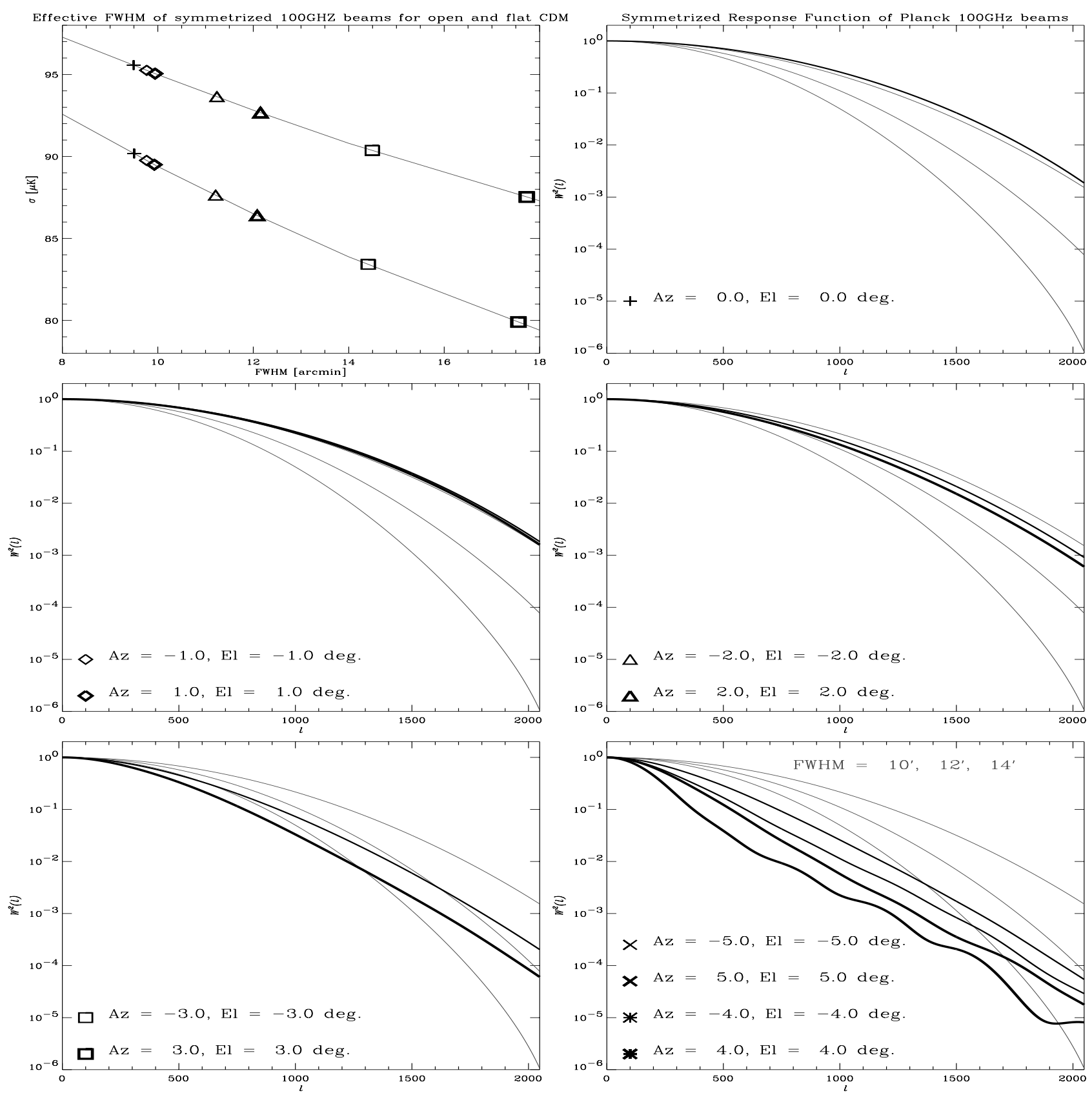

Fig. 13. The same as Fig. 6 but for the BAFFLE.1550 configuration

Table 1. Parameters of telescope designs analyzed

\begin{tabular}{ccccccc}
\hline Design & $D_{\mathrm{p}}(\mathrm{mm})$ & $F_{\mathrm{p}}(\mathrm{mm})$ & $2 a(\mathrm{~mm})$ & $2 c(\mathrm{~mm})$ & $d_{\mathrm{m}-\mathrm{s}}(\mathrm{mm})$ & $f_{\text {eff }} / D$ \\
\hline BAFFLE.1550 & 1550.0 & 720 & 1200 & 514.29 & 1065.69 & 1.08 \\
BAFFLE.1750 & 1750.0 & 720 & 1200 & 514.29 & 1065.69 & 1.08 \\
\hline
\end{tabular}



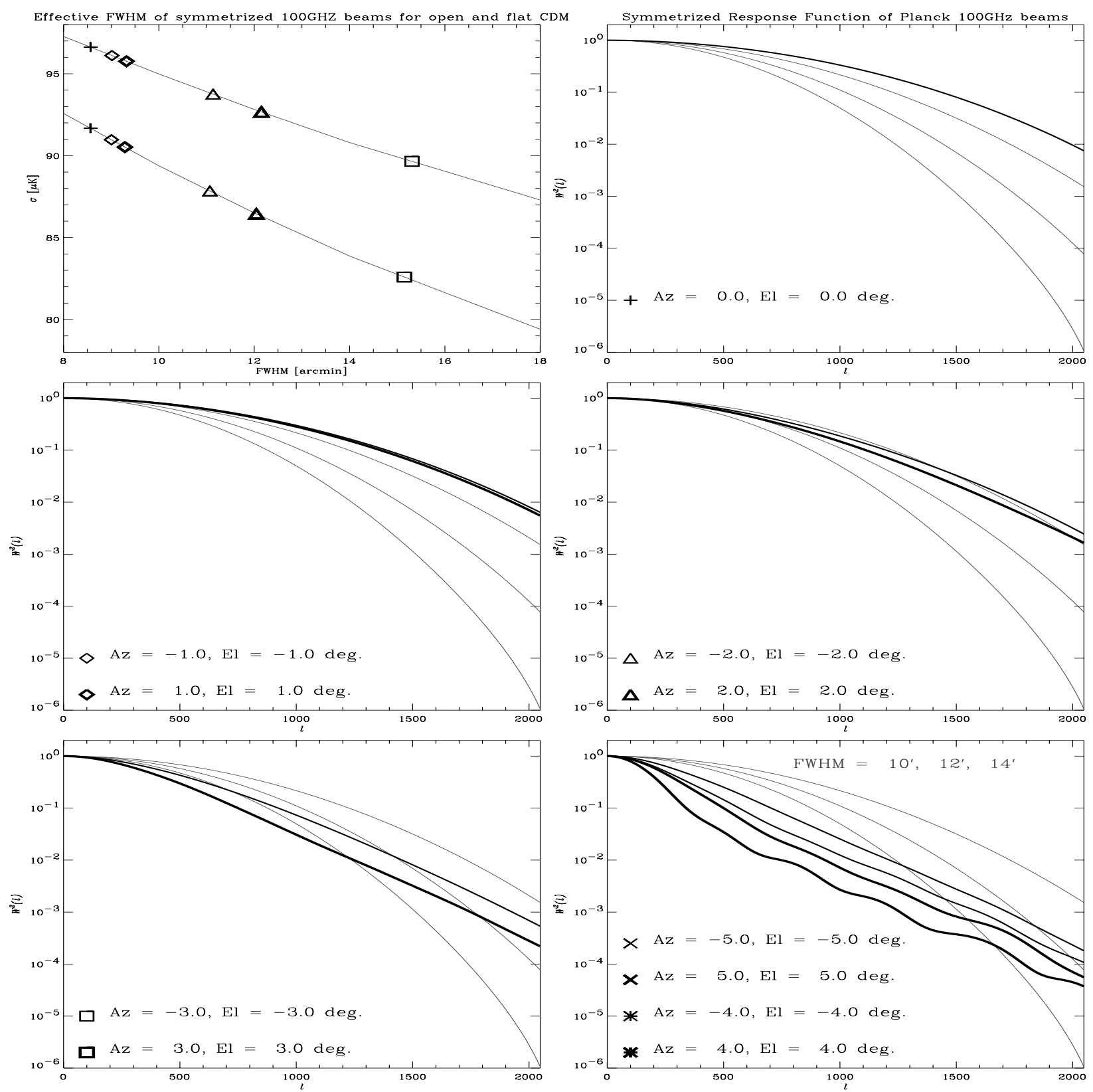

Fig. 14. The same as Fig. 6 but for the BAFFLE.1750 configuration

The comparison with the PHASE A telescope shows an improvement in the angular resolution restricted to regions very close to the optical axis and therefore not relevant for all (LFI and HFI) receivers, which are located at the same relevant distance from the centre as in the case of the PHASE A telescope. These options do not allow to reach then the crucial goal of $10^{\prime}$ resolution at $100 \mathrm{GHz}$.

\section{Appendix B: Beam optical properties of the $30 \mathrm{GHz}$ beams}

As well known, for the same telescope and feed position, the beam optical distortions increase with the frequency.
On the other hand, we know that for example for elliptical beams with the same eccentricity the difference of the observed temperature with respect to the case of symmetric beams increases with the beam size (Burigana et al. 1998). Whereas the HFI feeds are located close to the optical axis, particularly at the highest frequencies, the distance from the telescope optical axis of the LFI feeds increases with the wavelength. It is then crucial to analyze the optical performance of PLANCK telescope also at the lowest frequency, the $30 \mathrm{GHz}$ channel.

In this case we have only two beams with identical optical properties, due to the symmetry of their positions in the FPU. By exploiting the Method 3, only considering the sky convolution with the simulated beam and with 

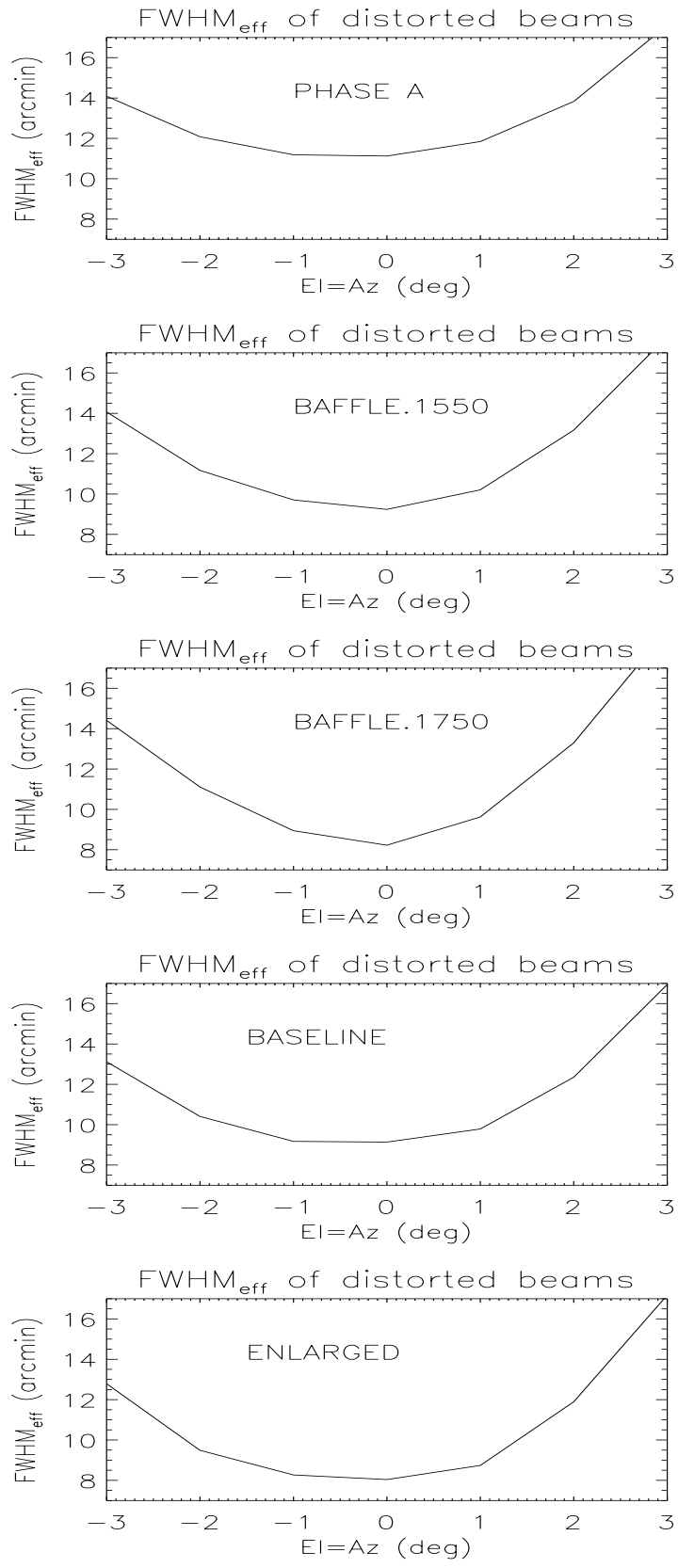
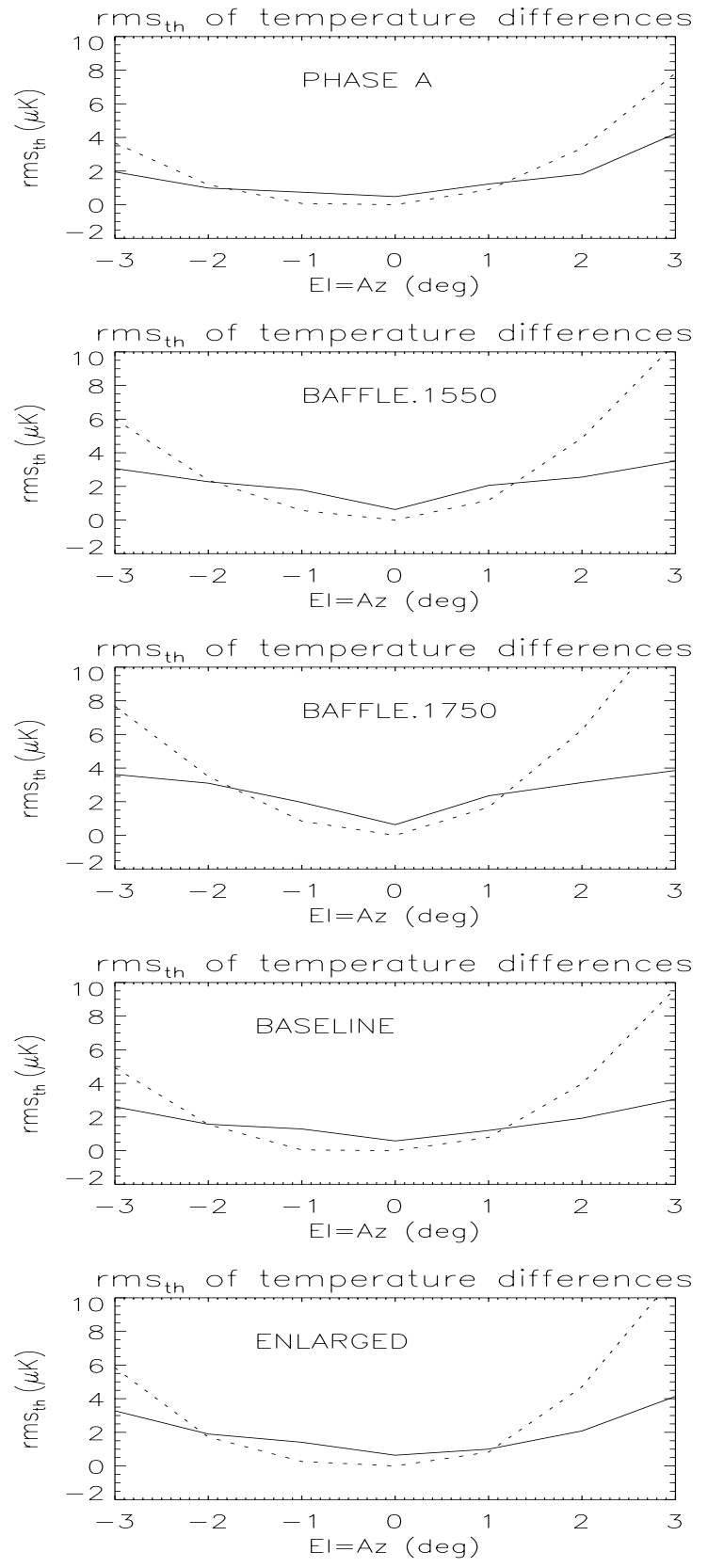

Fig. 15. Synthetic analysis of simulated beams based on the Method 3 for all the considered telescope designs. Left panels: effective width, $F W H M_{\text {eff }}$, of distorted beams a function of their elevation and azimuth. Right panels: rms th of the temperature differences between observations performed by a distorted beam and the corresponding symmetric Gaussian one (solid line) and between observations performed by a Gaussian beam with the same $F W H M$ of the distorted one and a Gaussian beam with the same FWHM of the central "real" beam (dotted line). (Channels at $100 \mathrm{GHz}$ )

Gaussian beams of comparable width up to $3^{\circ}$ from the beam centre (and using then a suitable larger number of grid points, namely $96 \times 96$ grid points) we estimated its equivalent $F W H M$ for the PHASE A and ENLARGED telescope: we find $38.86^{\prime}$ and $28.72^{\prime}$ respectively. Also, the corresponding rms temperature difference between the distorted beams and the corresponding Gaussian beams is $\simeq 2 \mu \mathrm{K}$ and $\simeq 1.2 \mu \mathrm{K}$ respectively.

Again, also at low frequencies a larger telescope significantly reduces the impact of beam distortions and makes much more comfortable to reach a good compromise between the reduction of sidelobe contamination and a good resolution. 


\section{References}

Bedini L., et al., 1999, "Image deconvolution and source separation", contribution to the "PlANCK LFI Consortium Meeting", Capri, October 7-9, 1999

Bersanelli M., et al., 1996, ESA, COBRAS/SAMBA Report on the Phase A Study, D/SCI(96)3

Bond J.R., Efstathiou G., Tegmark M., 1997, MNRAS 291, L33

Bouchet F., et al., 1995, Space Sci. Rev. 74, 37

Burigana C., et al., 1998a, A\&AS 130, 551

Burigana C., et al., 1998b, Proceedings of the XXXIII ${ }^{\mathrm{rd}}$ Rencontres de Moriond, "Fundamental Parameters in Cosmology", Les Arcs, France, January 17-24, 1998, Trân Thanh Vân et al. (eds.), p. 171

Burigana C., et al., 1999a, Astro. Lett. Comm. (in press) astro-ph/9903137

Burigana C., et al., 1999b, "Effects of instrumental noise and optical distortions on PLANCK LFI observations", contribution to the "PlANCK LFI Consortium Meeting", Capri, October 7-9, 1999

Danese L., et al., 1996, Astro. Lett. Comm. 35, 257

Delabrouille J., 1998, A\&AS 127, 555

De Maagt P., Polegre A.M., Crone G., 1998, Planck Straylight Evaluation of the Carrier Configuration, Technical Report ESA, PT-TN-05967, 1/0

De Zotti G., et al., 1999, Proceedings of the EC-TMR Conference "3 K Cosmology", Roma, Italy, 5-10 October 1998, AIP Conference Proc. 476, Maiani L., Melchiorri F., Vittorio N. (eds.), astro-ph/9902103, p. 204

Hobson M.P., et al., 1999, MNRAS 306, 232
Knox L., 1995, Phys. Rev. D 48, 3502

Maino D., et al., 1999, A\&AS 140, 1

Maino D., 1999, Ph.D. Thesis at SISSA

Mandolesi N., et al., 1997, Int. Rep. TeSRE/CNR 199/1997

Mandolesi N., et al., 1998, Planck LFI, A Proposal (submitted to the ESA)

Mandolesi N., et al., 1999, Astro. Lett. Comm. (in press) astro-ph/9904135

Puget J.L., et al., 1998, HFI for the Planck Mission, A Proposal (submitted to the ESA)

Puget J.L., Delabrouille J., 1999, "HFI Sidelobe Straylight Requirement Document" - 2nd Revision - March 16, 1999

Sletten C.J., 1988, Reflector and Lens Antennas - Analysis and Design Using Personal Computers, Artech House

Sanz J.L., et al., 1999a, MNRAS (in press) astro-ph/9906367

Sanz J.L., et al., 1999b, A\&AS (in press) astro-ph/9909497

Tegmark M., Efstathiou G., 1996, MNRAS 281, 1297

Tegmark M., et al., 1999, ApJ (submitted) astro-ph/9905257

Tenorio L., et al., 1999, MNRAS (submitted) astro-ph/9903206

TICRA Report 22-09-97 and 23-09-97, 1997 (communication at the Planck Telescope Working Group, Copenhagen, 26.9.1997)

Toffolatti L., et al., 1998, MNRAS 297, 117

Valenziano L., et al., 1998, Int. Rep. TeSRE/CNR 224/1998

Villa F., et al., 1997, Int. Rep. TeSRE/CNR 193/1997

Villa F., Mandolesi N., Burigana C., 1998a, Int. Rep. TeSRE/CNR 221/1998

Villa F., et al., 1998b, Int. Rep. TeSRE/CNR 231/1998

Wandelt B.D., Górski K.M., 1999, "A Preliminary Study of Sky Observations with the PLANCK Surveyor Satellite", http://www.tac.dk/ ${ }^{\sim}$ wandelt/papers.html 